\title{
Is the large angle CMB Inconsistent with concordance cosmology?
}

\author{
Glenn Starkman* \\ Dept of Physics/CERCA/ISO, Case Western Reserve University \\ Cleveland, OH 44106-7079 USA \\ E-mail: glenn.starkman@case.edu
}

\section{Craig J. Copi}

Dept of Physics/CERCA/ISO, Case Western Reserve University

10900 Euclid Ave, Cleveland, OH 44106-7079 USA

E-mail: cjc5ecase.edu

\section{Dragan Huterer}

Department of Physics, University of Michigan,

450 Church St, Ann Arbor, MI 48109 USA

E-mail: huterereumich.edu

\section{Dominik Schwarz}

Fakultät für Physik, Universität Bielefeld,

Postfach 100131, 33501 Bielefeld, Germany

E-mail: dschwarzaphysik.uni-bielefeld.de

The measured fluctuations in cosmic microwave background radiation is one of the principal tools for studying cosmology. It is widely portrayed as providing extraordinary confirmation of the standard model of cosmology - inflationary $\Lambda$ Cold Dark Matter - and is the single most important data set for determining the parameters of that model. However, the large-angle/low-1 portion of the CMB fluctuations, as measured most recently and completely by the Wilkinson Microwave Anisotropy Probe, display features that are at odds with the standard model. The fully-reconstructed sky demonstrates a peculiar alignment of the quadrupole and the octopole components of the fluctuations with each other. Moreover, those aligned multipoles define a plane perpendicular to the ecliptic plane, and apparently pointing toward the CMB dipole. The ecliptic rather precisely separates the strong extrema of the southern ecliptic sky from the weaker extrema of the northern ecliptic sky. If the reconstructed sky behind the galaxy is excluded, the CMB demonstrates an unusual lack of correlation for pairs of directions separated by more than $60^{\circ}$. This lack of correlation is unlikely to be present in a universe which is a realization of Gaussian random statistically isotropic modes proportional to spherical harmonics, as is predicted by the standard model.

International Workshop on Cosmic Structure and Evolution - Cosmology2009,

September 23-25, 2009

Bielefeld, Germany

\footnotetext{
* Speaker.
} 
When we look at the sky in the microwave band, we have, since Penzias and Wilson in the 1960 s, seen an isotropic glow characterized by an antenna temperature of about $3^{\circ} \mathrm{K}$. This glow is the relic radiation of a time when the universe was younger, denser and hotter - about $3000^{\circ} \mathrm{K}$, comparable to the surface temperature of a star. Its discovery was therefore confirming evidence of the Big Bang theory of cosmology - the theory that we live in a universe that is expanding and cooling from an initially hot dense state.

We've also known, pretty much since then, that that cosmic microwave radiation ought not to be perfectly homogeneous, After all, the universe today is awfully lumpy - as we can see by looking around the room - and that lumpiness should be evident even in this fossil record of the early universe. Indeed, the most reasonable explanation for the current inhomogeneity of the universe is that it developed through the action of gravity from earlier primordial inhomogeneities. These seeds of structure should be evident in the CMB.

The road to discovery of the CMB anisotropies was longer and harder than was first anticipated. A dipolar anisotropy caused, presumably, by the Doppler effect due to Earth's motion through the universe, was discovered in the 1970s; but it took until the Cosmic Background Explorer (COBE) in the early 1990s for compelling evidence of primordial anisotropies - a discovery significant enough to earn the project leaders the 2006 Nobel Prize in Physics.

The modern standard in CMB anisotropies is the Wilkinson Microwave Anisotropy Probe (WMAP). This satellite, launched in 2001, provides, at least for the next few months when it is likely to be displaced by the Planck satellite, the best full sky maps of the CMB. In this talk I will discuss what we can learn about the largest angle properties of the CMB from WMAP data. We will identify the original low-1 problem - the smallness of the quadrupole component of the angular power spectrum. Motivated by that, we will examine the more detailed structure of the quadrupole and octopole, discovering that they seem unexpectedly aligned with each other and with physical directions or structures on the sky that are associated with the WMAP experiment. Finally, we will return to the low-l problem and realize that it is far more serious when viewed in angle-space instead of 1-space, and that it presents a serious challenge to the absolutely canonical prediction of the standard cosmological model - inflationary $\Lambda \mathrm{CDM}$ - of statistically independent $C_{l}$ 's.

One of the first lessons in cosmology kindergarten is that when presented with a continuous function on the sphere of the sky (as opposed to a catalogue of objects) one should expand that function in spherical harmonics. This applies to the WMAP full sky maps that are synthesized by various groups from the individual band maps released by the WMAP team in an attempt to faithfully reconstruct the CMB temperature over the full sky. We shall focus our attention on the WMAP team's own Internal Linear Combinations map [1], although other maps (LILC, TOH, NILC [2]) are available. These results are only slightly dependent on the choice of maps.

We thus write for the temperature fluctuations, $\Delta T$

$$
\Delta T(\theta, \phi)=\sum_{l, m} a_{l, m} Y_{l, m}(\theta, \phi),
$$

where the sum is generally taken over $l \geq 2$, since the dipole is dominated by the Doppler contribution due to the motion of the Sun and the satellite through the microwave background. The angular power spectrum is

$$
C_{l}=\frac{1}{2 l+1} \sum_{m}\left|a_{l, m}\right|^{2}
$$


The $a_{l m}$ are predicted by inflationary $\Lambda \mathrm{CDM}$ to be (very nearly) independent Gaussian random variables, and to be statistically isotropic. This means that the ensemble average of $a_{l m}$ obeys:

$$
\left\langle a_{l, m} a_{l^{\prime}, m^{\prime}}^{*}\right\rangle=\delta_{l l^{\prime}} \delta_{m m^{\prime}} C_{l} .
$$

The expected values of $C_{l}$ are predicted by the theory.

It is by now well known that the agreement between the observed WMAP $C_{l}$ and a smalldimensional parameterization of the predicted $C_{l}$ by $\Lambda \mathrm{CDM}$ is remarkable. One of the first things we learn is that the universe is very close to a flat geometry. Indeed the radius of curvature of the geometry is approximately $3000 h^{-1} M p C / \sqrt{1-\Omega}$, where $h^{-1} \simeq 0.72$, and $1.0179>1-\Omega<0.992$ at the $95 \%$ CL [3].

Is there anything else to learn about the universe on large scales?

The first thing that suggested that there may be was the observation already by the Cosmic Background Explorer (COBE) team in the early 1990s that the quadrupole $\left(C_{2}\right)$ is anomalously low. This observation was confirmed by WMAP. One thing that this observation spawned was a renewed interest in cosmic topology - the possibility that the universe in which we live has closed curves that cannot be smoothly collapsed to a point. Or, as some prefer to put it, the possibility that we live in a small or compact universe.

As an explanation for the smallness of the quadrupole, the argument for the utility of a compact universe is that if the universe is compact, then modes with wavelength larger than the compactification scale cannot be supported by the space. This reduction in the number of large scale modes will result in a reduction of the large scale power. Unfortunately this argument is flawed for many reason, not the least of which is that it relies on an assumption that all modes are populated with equal amplitudes by inflation. However, if we are seeing the full extent of the universe then inflation makes no reliable predictions for the amplitude of modes of that wavelength.

Nevertheless, the possibility of cosmic topology is a legitimate one. And it certainly could lead to unexpected values for low-l $C_{l}$. While there have been some claims of discovery of cosmic topology, they are not widely accepted. The best reported limits on it require that the shortest closed null geodesic be longer than $24 \mathrm{GpC}$ [4], just shy of the diameter of the horizon. Of course, topology could affect the low-l $C_{l}$ even though the scale of topology is larger than the horizon diameter. This would make this explanation that much more difficult to test.

Whatever the explanation for the low quadrupole, we are motivated to look beyond just the angular power spectrum to see if the low-l multipoles are otherwise well behaved. In fact, many anomalies have been noted with regard to the low-1/large-angle properties of the CMB. We shall not list them here, not because they are not interesting, but because they are too numerous for this space. Suffice it to say that each in its own way retells some of the ensuing story. The particular set of anomalies that I would like to discuss grew out of the search for topology beyond the horizon. In analogy with the interchangeability of the description of $l=1$ in terms of $a_{1 m}(m=-1,0,1)$ with a description in terms of a dipole vector, we sought to describe the 1-th multipole by special directions, with the idea that somehow the topology would correlate the directions of different multipoles. With this in mind we unwittingly reinvented [5] an old idea dating back at least to Maxwell of multipole vectors [6].

One can show that the $2 l+1 a_{l m}$ of any given $l$, can be replaced by $l$ unit vectors and a single scalar. These unit vectors (whose sign can be absorbed into the scalar) are termed multipole vectors. 


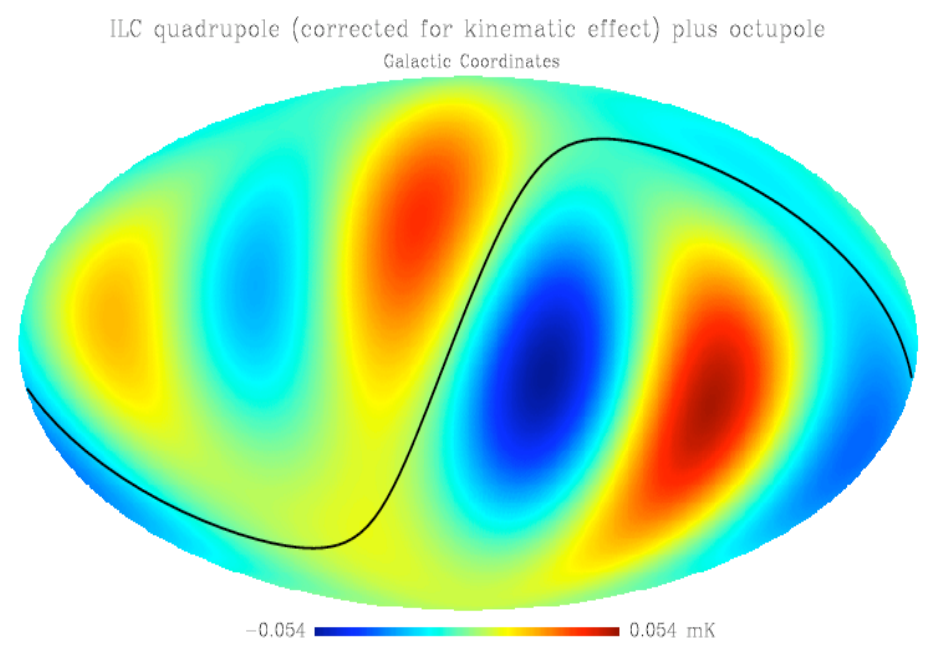

Figure 1: The $l=2$ and 3 multipoles. The solid curve is the ecliptic plane.

Their statistical properties have been calculated by Dennis et al. [7, 8] under the assumption that the $a_{l m}$ are Gaussian random and statistically isotropic. Certainly, however, the multipole vectors of different $l$ should be uncorrelated.

The $5 a_{2 m}$ of the quadrupole are thus replaced by $C_{2}$ and by a pair of multipole vectors. (Or, more accurately by a scalar $A_{2}$ which depends on $C_{2}$, and a pair of multipole vectors which are independent of $C_{2}$.) The quadrupole can thus be thought of as a plane. Similarly, the $7 a_{3 m}$ describing the octopole can be replaced by $C_{3}$ and by three multipole vectors. The octopole can therefore be thought of as three planes. The three planes of the octopole and the plane of the quadrupole when calculated for the ILC map turn out to be surprisingly parallel to each other [9]. Indeed, the observed degree of alignment is seen in only about $0.1 \%$ of realizations of inflationary $\Lambda$ CDM [11]. This phenomenon was first described (albeit using other statistics, which we of course find less intuitive) in [10]. The normal to these planes was later termed the "Axis of Evil", a mild misnomer since there is no axial symmetry of the multipoles around their normal. Perhaps it should be called instead the Plane of Pain.

If these alignments among the planes of the octopole and the quadrupole were the end of the story then it might suggest that the explanation is some rather odd cosmological phenomenon. However, in [9] and the more detailed [11], we showed that these planes are surprisingly perpendicular to the ecliptic - the plane of the Earth's (and WMAP's) orbit around the Sun. The probability that this alignment would happen by chance, even given the unlikely alignment of the quadrupole and octopole planes, was at most $2 \%$. Furthermore, the plane is oriented in such a way that its normal points surprisingly close to the dipole - the direction of the Sun's motion through the universe (relative to the CMB) - which just happens to lie near the ecliptic. Finally, to a very good approximation, the quadrupole plus octopole can be described as a ring (defined by this common plane) of six extrema that are evenly spaced around the ring. This ring of six is so positioned that the ecliptic very carefully threads its way between them, thus separating the three strongest extrema from the three weakest. The picture of this (Figure 1) is rather remarkable.

It is difficult to understand how cosmological physics could have produced these alignments 
with the solar system, except by some unkind coincidence. But it is no less difficult to understand how foregrounds could have done it. In particular if the aligned signal is due to the foreground, then the CMB signal must be smaller than the foreground. This in and of itself would be a surprise. Moreover, the multipole structure of the observed quadrupole and octopole is aligned planar multipoles - ie. $Y_{l l}$ with the appropriate choice of z-axis. Explanations which yield aligned $Y_{l 0}$ are not adequate. Finally, the observed power in the octopole is comparable to the power in the quadrupole. Therefore explanations which involve expansions in vectors of small magnitude are unlikely to succeed if the octopole results from a higher order in the expansion than does the quadrupole.

Having identified the aforementioned mysterious alignments, let us return to the original observation of a small quadrupole and ask whether that is really the salient feature in the low-l angular power spectrum. A hint to the answer to that question was provided by one of the later COBE-DMR papers [12]. Back in those times, it was not yet unusual to plot, not just the angular power spectrum, but the two point correlation function

$$
\left.C(\theta) \equiv \overline{T\left(\Omega_{1}\right) T\left(\Omega_{2}\right)}\right|_{\Omega_{1} \cdot \Omega_{2}=\cos \theta} .
$$

(The overbar indicates a sky average over all pairs of points separated by an angle $\theta$.)

$C(\theta)$ is closely related to $C_{l}$. On a full sky they contain the same information, and $C(\theta)=$ $\Sigma(2 l+1) C_{l} P_{l}(\cos \theta)$. However, one should be cautious in jumping to the conclusion that therefore $C_{l}$, which offers a more direct window into inflationary theory, is always the correct set of variables. This would be analogous to saying that one should always look at some time series in the Fourier domain, never in the time domain.

The authors of [12] noted that the two point correlation function seemed to be unexpectedly close to zero above about $60^{\circ}$. However, this anomaly was not terribly statistically significant. Had they done a statistical analysis (which they do not seem to have done), they would have found that the correlation function at large angles remains this close to zero in about $5 \%$ of realizations of our now-concordance cosmology. The WMAP team confirmed this anomaly in the papers describing their first year of observations. The anomaly, as quantified by the statistic they proposed,

$$
S_{1 / 2}=\int_{-1}^{1 / 2}(C(\theta))^{2} d \cos \theta
$$

was observed to be smaller than in approximately $0.1 \%$ of random realizations of $\Lambda \mathrm{CDM}$ [13].

Since then, the anomaly has appeared to largely go away in the reported WMAP results. However, we have shown [15] that this reflects largely a change in what is reported. If, as in the first year WMAP analysis, one extracts the angular correlation function directly from the part of the sky outside the galaxy (for example outside the KQ75 mask), then one finds that $C(\theta)$ in the five year WMAP data is largely unchanged from the first year data. Indeed, in the ILC, if one calculates $C(\theta)$ over the whole sky, one finds that almost all the contribution above $60^{\circ}$ comes from pairs of points at least one of which is inside the KQ75 galaxy mask. $S_{1 / 2}$ measured outside that mask is so small that only in about $0.03 \%$ of random realizations of $\Lambda \mathrm{CDM}$ with the standard parameter values does it come out smaller. This is discussed at length in [14] and [15], which also show how this is unlikely to be an accident of the galaxy happening to overly the power in the quadrupole and octopole. It is also showed that the smallness of $S_{1 / 2}$ is not equivalent to the smallness of $C_{2}$. 
Indeed, it appears to require the conspiracy of $C_{2}, C_{3}, C_{4}$, and $C_{5}$ - in which the contributions from those multipoles cancel those from higher multipoles - to explain how little large angle two point correlation function there actually is.

Perhaps the most intriguing aspect of this is that the small two-point correlation function cannot be obtained merely by changing the theoretical $C_{l}$. The reason is that even if we change the theoretical $C_{l}$ by hand to make the expected $C(\theta)$ very close to zero, the process of drawing gaussian $a_{l m}$ with variance $C_{l}$ will yield measured $C_{l}$ sufficiently different from the theoretical expectations so as to reduce the precise cancellations that go into getting a very small $C(\theta)$. Thus a small $S_{1 / 2}$ challenges a most fundamental prediction of standard cosmology - that the $a_{l m}$ are Gaussian random and statistically isotropic (GRSI). So far this is a 98\% C.L. statement, but ongoing work suggests that GRSI may be ruled out at much greater confidence. If so, our standard model of cosmology will demand even more strongly some modification on large scales.

\section{References}

[1] The WMAP team's Internal Linear Combinations map is available from NASA's Legacy Archive for Microwave Background Data Analysis at http://lambda.gsfc.nasa.gov/product/map/dr3/ilc_map_get.cfm

[2] ILC: Eriksen et al. Astrophys.J. 612 (2004) 633-646; TOH: M. Tegmark, A. de Oliveira-Costa and A. Hamilton, PRD 68, 123523 (2003); NILC: J. Delabrouille et al. A\&A 493, 835-857 (2009) .

[3] E. Komatsu et al. [WMAP Collaboration], Astrophys. J. Suppl. 180, 330 (2009) [arXiv:0803.0547 [astro-ph]].

[4] J. Shapiro Key, N. J. Cornish, D. N. Spergel and G. D. Starkman, Phys. Rev. D 75, 084034 (2007) [arXiv:astro-ph/0604616].

[5] C. J. Copi, D. Huterer and G. D. Starkman, Phys. Rev. D 70, 043515 (2004) [arXiv:astro-ph/0310511].

[6] James Clerk Maxwell, A Treatise on Electricity and Magnetism, 1st ed. 1873, 3rd ed. 1891, reprinted by Dover Publications, 1954.

[7] M. R. Dennis and K. Land, arXiv:0704.3657 [astro-ph].

[8] M. R. Dennis, J. Phys. A 38, 1653 (2005) [arXiv:math-ph/0410004].

[9] D. J. Schwarz, G. D. Starkman, D. Huterer and C. J. Copi, Phys. Rev. Lett. 93, 221301 (2004) [arXiv:astro-ph/0403353].

[10] A. de Oliveira-Costa, M. Tegmark, M. Zaldarriaga and A. Hamilton, Phys. Rev. D 69, 063516 (2004) [arXiv:astro-ph/0307282].

[11] C. J. Copi, D. Huterer, D. J. Schwarz and G. D. Starkman, Mon. Not. Roy. Astron. Soc. 367, 79 (2006) [arXiv:astro-ph/0508047].

[12] G. Hinshaw et al., Astrophys. J. 464, L25 (1996), astroph/ 9601061.

[13] D. N. Spergel et al., Astrophys. J. 148, S175 (2003),

[14] C. Copi, D. Huterer, D. Schwarz and G. Starkman, Phys. Rev. D 75, 023507 (2007) [arXiv:astro-ph/0605135].

[15] C. J. Copi, D. Huterer, D. J. Schwarz and G. D. Starkman, Mon. Not. Roy. Astron. Soc. 399, 295 (2009) [arXiv:0808.3767 [astro-ph]]. astro-ph/0302209. 